\title{
Virtual screening of FDA approved drugs against Nsp15 endoribonuclease from SARS CoV-2
}

Althaf Shaik*1, Nalini Natarajan², Sivapriya Kirubakaran1, Vijay Thiruvenkatam³

${ }^{1}$ Discipline of Chemistry, Indian Institute of Technology Gandhinagar, Palaj, Gandhinagar, Gujarat382355

2Discipline of Bioengineering, Indian Institute of Technology Gandhinagar, Palaj, Gandhinagar, Gujarat382355

${ }^{3}$ Discipline of Physics and Bioengineering, Indian Institute of Technology Gandhinagar, Palaj, Gandhinagar, Gujarat-382355

* Equal contribution

\#Corresponding author

Email: vijay@iitgn.ac.in

\section{Abstract}

COVID -19, a pandemic that has spread to more than 200 countries is caused by SARS-CoV2 (Coronavirus). Due to rapidly spreading capability of this virus, the entire world is fighting to mitigate the infection in an unprecedented way. Several researchers around the world are trying to treat COVID-19 by different strategies, which includes blood plasma treatment, anti-viral or anti-malarial drug trials. However, there has not been a proven cure identified for this infection yet. Different aspects of SARS-CoV2 are explored at a rapid pace in the laboratories throughout the world. For instance, the genomic and the protein structural information from the recent reports are valuable resources for further studies. It is also important to explore different druggable targets against the viral multiplication. Here, we have done virtual screening of 2910 FDA drugs against SARS-CoV2 by targeting non-structural protein 15 (Nsp15) endoribonuclease. We have identified the top 20 hits and have further analyzed their interaction and stability using Induced Fit Docking (IFD) and Molecular Dynamics (MD) simulations. Our results show that the top five drug hits or their scaffolds after careful experimental evaluation can be repurposed for the treatment of COVID-19.

Keywords: COVID-19, Nsp15, coronavirus, FDA drugs, molecular docking, virtual screening, antiviral drugs, antibacterial, anticancer, IFD 


\section{Introduction}

The outbreak which started in the late December 2019 eventually turned out to be the greatest pandemic of our generation is caused by a coronavirus named SARS-CoV2 (Severe acute respiratory syndrome coronavirus-2). The disease caused by the SARS-CoV2 is termed as COVID-19 by the world health organization (WHO). The outbreak was first reported in Wuhan, China in the December of 2019. Since then the virus has spread to 218 countries so far affecting more than 56 million people and have caused 1.3 million deaths till date. The mode of transmission of this virus according to the available findings includes droplet transmission from the infected person, duration in air as aerosols and on fomite. Over half of the world's populations are under social distancing, lockdowns, quarantines and isolation to mitigate the spread of infection. The time required in finding a way to counteract the infection is highly precious and finding a new drug and making it available in the market takes several years of laboratory work and multiple stages of clinical trials. Besides, the approval by FDA after all the necessary studies takes about six months to one year. Therefore, the scientists around the world are focusing on repurposing the medications that are pre-approved by FDA for treating other ailments. Nevertheless, it is inevitable to understand the mechanistic, structural and functional elements of SARS-CoV2 for a permanent cure of this disease in the future. Fortunately, the scientific community around the world are unravelling the aspects of this virus at a rapid pace. This eases the process of getting a quick understanding of the virus and use the pre-existing FDA approved drugs with better precision to curb the infection.

SARS-CoV2 belongs to the nidovirales order, Coronaviridae family, Sarbecovirus subgenus and Betacoronavirus genus ${ }^{1}$. The whole genome sequencing has phylogenetically shown that the SARS-CoV2 is closer to bat-SL-CoVZC45 and bat-SL-CoVZXC21 than the SARS-CoV. However, at a protein level SARS-CoV2 shares the highest homology towards SARS-CoV². 
Despite the whole genome similarity of only $79 \%$ between that SARS-CoV and SARS-CoV2, some of their proteins share a higher similarity percentage like RdRP (RNA dependent RNA polymerase) and 3CLpro (coronavirus main protease), which are about $95 \%$ similar ${ }^{3}$. Coronaviruses structure involves an outer lipid membrane in which the spike proteins are embedded to the membrane which gives characteristic name for this family of viruses (coronacrown). Inside the membrane the virus genetic material consists of positive RNA which is about $39 \mathrm{~Kb}$. The spike protein (S-protein) of SAR-CoV2 has two domains S1 and S2. The recent cryo-EM structure of SARS-CoV2 shows that the S-protein binds to the Angiotensin-converting enzyme-2 (ACE-2) ${ }^{4}$. The surface plasmon resonance interaction of the S-protein to ACE-2 have also shown that SARS-CoV2 may bind 10 times stronger than the SARS-CoV ${ }^{5-6}$.

The viral genome consists of several non-structural proteins (Nsps) most of which aid in RNA replication and sub genomic RNA processing. Some of the Nsps are proteases that helps in cleaving the encoded polypeptide into several non-structural proteins that perform different functions in the viral replication. The two proteases include 3C-like protease (3CLpro) encoded by Nsp5 and papain-like protease (PLP), which is encoded by Nsp37. There are in total 16 Nsps and functions of some of these proteins are not completely understood. Nsp15 is an endoribonuclease that acts on both single and double-stranded RNA ${ }^{8}$. Nsp15 comes under nidovirus endonucleases (NendoU), which specifically targets uridylates in RNA to form 2'-3'cyclic phosphodiester products ${ }^{9}$. The virus employs a defense mechanism by protecting its dsRNA (during the RNA synthesis) by hiding it in a double membrane vesicle (DMV) inside the host cell ${ }^{10}$. Nsp15 has a role to play in formation of DMV and thereby protecting the viral dsRNA from the host dsRNA sensors that could trigger the host's immune response ${ }^{11}$. Amongst the Nsp15s from other viruses SARS-CoV Nsp15 was the first reported to have NendoU activity in vitro $^{12-13}$. The catalytic site of NendoU and its other homolog from Xenopus origin XendoU consists of a catalytic triad including two histidine and one lysine residue ${ }^{14}$. 
Although the vaccines and antibody treatments are very effective, they are expensive, laborious and are still at a risk of being obsolete if the virus mutates. Recent reports have shown that the spike protein, against which many vaccines are developed, has shown several mutations ${ }^{15}$. Hence, diverse treatment options focusing on different protein targets in SARS-CoV2 should be considered. In our study, we have virtually screened 2910 FDA approved drugs against Nsp15 and have shown few hits with high binding affinity. We have also studied the stability of these drugs' interaction with Nsp15 using both Induced fit docking (IFD) and molecular dynamic (MD) simulations. We propose that our top drug hits can serve by itself or can help in design of novel chemical scaffolds as a treatment of COVID-19.

\section{Active site NendoU (Nsp15)}

The catalytic site of Nsp15 consists of two anti-parallel beta-sheets $(\beta 16-\beta 17-\beta 18$ and $\beta 19-\beta 20-$ $\beta 21)$ at the C-terminal of the protein. In the solution, Nsp15 is found to be active in the form of hexamer (dimer of trimer). Nsp15 requires $\mathrm{Mn}^{2+}$ to cut the double stranded RNA at the $3^{\prime}$ uridylates forming 2'-3' cyclic phosphodiester product ${ }^{12,}{ }^{16}$. The active site consists of six key residues HIS235, HIS250, LYS290, THR341, TYR343, and SER294, which are conserved in SARS-CoV, MERS-CoV and SARS-CoV2. The structural arrangement of these amino acids is also conserved in all three proteins except for LYS290. HIS235, HIS250 and LYS290 are the catalytic triad as it closely represents the active site of ribonuclease family of proteins ${ }^{17}$.

\section{Materials and methods}

The atomic coordinates of the Nsp15 endoribonuclease from SARS CoV-2 $2^{17}$ (PDB ID: 6VWW) was retrieved from the Protein Data Bank ${ }^{18}$ and was used for the molecular docking and simulation studies. It has 346 amino acids with a resolution of 2.20A. 2910 FDA approved compounds in the .sdf format were retrieved from the ZINC database ${ }^{19}$ and were screened against Nsp15 endoribonuclease to determine initial hits or scaffolds. 


\subsection{Ligand and Protein preparation}

The retrieved FDA ligands were subjected to ligand preparation using Ligprep module in Maestro (Schrödinger, LLC, New York, USA, 2018-4) to generate various conformations, correction, verification and optimization of the structures. The protein preparation wizard in Maestro (Schrödinger, LLC, New York, NY, 2018-4) was used to prepare and optimize the protein for molecular docking studies. The retrieved Nsp15 Endoribonuclease from SARS CoV2 protein was subjected to protein preparation protocol to add polar hydrogens, $\mathrm{pH}$ adjustments, optimization and minimization using OPLS2005 force field.

\subsection{Grid generation and Docking}

Receptor grid was generated using Receptor grid generation module in the Glide (Glide, v8.1, Schrödinger, LLC, New York, USA, 2018-4) of Maestro suit (Schrödinger, LLC, New York, USA, 2018-4). A $20 \AA ̊$ receptor grid for Nsp15 endoribonuclease from SARS CoV-2 was generated by specifying the catalytic site residues (HIS235, HIS250, LYS290) ${ }^{17,20}$. Once the receptor grid was generated, the FDA drugs were docked into the Nsp15 active site using "High throughput virtual screening protocol” (HTVS) in Glide v8.1 (Grid based Ligand Docking with Energetics) docking protocol of Maestro suit (Schrödinger, LLC, New York, USA, 2018-4). The docked conformers were evaluated using Glide (G) Score ${ }^{21}$. Finally, from the HTVS screening we have identified best drugs based on the docking score and proceeded to Induced fit docking (IFD).

\subsection{Induced fit docking}

Induced fit docking approach was performed by using IFD protocol in Maestro suit (Schrödinger, LLC, New York, USA, 2018-4). IFD considers the conformational perturbations that are brought about by the ligands. It helps in creating various ligand poses with the possible binding modes and each of which is refined and ranked by prime. The top 20 ligands were redocked into the active site of the protein using reduced van der Waals radii (vdw) using glide XP mode. In order 
to account for the protein flexibility, the vdw of nonpolar atoms in the receptor were adjusted for scaling factor of 0.8 . The partial charge cut off was given as 0.15 , which ensures the scaling of the nonpolar atoms only. The side chain conformation and amino acid residue within $5.0 \AA$ of Nsp15 -ligand poses were refined by Prime energy. Finally, energy minimization was performed using OPLS-2005 force field for ranking the best posed in IFD.

\subsection{Molecular dynamic simulation}

Molecular dynamic simulations for docked complexes were performed using Desmond package (D.E. Shaw Research 2018) to understand the stability of predicted docked complex. Top 7 compounds that survived the Glide XP and IFD docking were subjected to MD simulation using TIP3P water molecules in an orthorhombic box. Nose-Hoover thermostatic and Martina-TobiasKlein was used for maintaining the constant temperature and constant pressure

The OPLS-2005 force field was used for minimization and the analysis of protein interactions. A grid spacing of $0.8 \AA$ in Particle-mesh Ewald (PME) method was used to calculate electrostatic interactions. Further, we have included the RESPA integrator with a near time step of 2.0 fs and far time step of 6.0 fs for bonded and non-bonded interactions. After system builder, all the complexes were subjected to the production run for $10 \mathrm{~ns}$ in the NPT ensemble at $300 \mathrm{~K}$ temperature and 1.013 bar pressure

\section{Results and discussion}

\subsection{High throughput virtual screening}

The virtual screening results of 2910 FDA drugs against Nsp15 endoribonuclease from SARS CoV-2 is presented in supplementary files. The top 20 drugs with highest docking score in throughput virtual screening are listed in Table 1. The highest dock score was of ceftolozane $(-7.83 \mathrm{kcal} / \mathrm{mol})$, an antibacterial drug that targets gram negative bacteria and is currently used for treating urinary tract infections. The lowest docking score cut off for our study was chosen to 
be $-5.50 \mathrm{kcal} / \mathrm{mol}$. Sofosbuvir, an anti-viral drug (hepatitis $\mathrm{C}$ virus protease inhibitor) was docked with the score of $-5.51 \mathrm{kcal} / \mathrm{mol}$, which was last entry included for our further analysis. Amongst the 20 drugs, five are associated with treating heart diseases (Dexrazoxane, Piceid, Cangrelor, Nordefrin and Metaraminol), five are anti-bacterial agents (Ceftolozane, Capastat, Nitrofurantonin, Amikacin and Plazomicin), four are anti-viral agents (Saquinavir, Paritaprevir, Dolutegravir and Sofosbuvir) two are anti-fungal (Natamycin and Ciclopirox) and two are anticancer (Azacitidine, and Oteracil). Interestingly, azacytidine, an anti-cancer drug and a potential anti-retro viral drug against HIV $22-23$, has given a docking score of $-6.74 \mathrm{kal} / \mathrm{mol}$ against Nsp15. The clinical applications of these top 20 compounds are listed in the table 1. Apart from the above drugs, certain cofactors (Flavin adenine mononucleotide (FAM), flavin adenine dinucleotide (FAD) and diphosphopyridine nucleotide (Dpnh) and hormones inhibitors (Atosiban) has also shown better affinity towards Nsp15 catalytic site.

Table 1. List of top 20 compounds in high throughput virtual screening (HTVS) of FDA drugs against NSP15.

\begin{tabular}{|c|c|c|c|c|}
\hline S. no & ZINC ID & Name & $\begin{array}{l}\text { HTP score } \\
\text { (kcal } / \mathrm{mol})\end{array}$ & Clinical application \\
\hline 1 & ZINC000009164421 & Ceftolozane & -7.83 & $\begin{array}{l}\text { Targets gram negative bacteria. Treats urinary tract infections, } \\
\text { intra-abdominal infections and ventilator-associated } \\
\text { bacterial pneumonia. }{ }^{24-25}\end{array}$ \\
\hline 2 & ZINC000016929327 & Azacitidine & -6.74 & $\begin{array}{l}\text { An anti-cancer drug: cytosine analogue }{ }^{26} \text {. Incorporates in DNA } \\
\text { and RNA. Used against HIV } 22 \text { and human T-Iymphotropic virus }{ }^{23}\end{array}$ \\
\hline 3 & ZINC000072190218 & Avatrombopag & -6.49 & low platelets associated with liver disease ${ }^{27}$ \\
\hline 4 & ZINC000087515509 & Dexrazoxane & -6.38 & $\begin{array}{l}\text { protects heart against cardiotoxic side effects from chemotherapy } \\
\text { drugs }^{28} \text { (EDTA analogue) and a potential anti-malarial }{ }^{29}\end{array}$ \\
\hline 5 & ZINC000013514753 & Oteracil & -5.99 & anti-cancer $^{30}$ \\
\hline 6 & ZINC000004098633 & Piceid & -5.97 & Anti-cancer ${ }^{31}$, anti-inflammatory, cardiovascular protection ${ }^{32}$ \\
\hline 7 & ZINC000085537017 & Cangrelor & -5.9 & Treats heart disease: platelet inhibitor ${ }^{33}$ \\
\hline 8 & ZINC000253633751 & Natamycin & -5.89 & Anti-fungal; binds to ergostero| ${ }^{34}$ \\
\hline
\end{tabular}




\begin{tabular}{|c|c|c|c|c|}
\hline 9 & ZINC000068150640 & Plazomicin & -5.86 & $\begin{array}{l}\text { Aminoglycoside antibiotic }{ }^{35} \\
\text { Treats urinary tract infection }\end{array}$ \\
\hline 10 & ZINC000113149554 & Netarsudil & -5.84 & Treats glaucoma or ocular hypertension ${ }^{37}$ \\
\hline 11 & ZINC000000034157 & Nordefrin & -5.8 & vasoconstrictor agent \\
\hline 12 & ZINC000026664090 & Saquinavir & -5.767 & HIV protease cleavage site analog ${ }^{38-39}$ \\
\hline 13 & ZINC000000001695 & Metaraminol & -5.71 & Increases blood pressure ${ }^{40}$ \\
\hline 14 & ZINC000008214483 & Amikacin & -5.69 & Aminoglycoside antibiotic, treats urinary tract infection ${ }^{41}$ \\
\hline 15 & ZINC000203757351 & Paritaprevir & -5.68 & Hepatitis C Virus protease inhibitor ${ }^{42}$ \\
\hline 16 & ZINC000058581064 & Dolutegravir & -5.64 & HIV - anti-retro viral ${ }^{43}$ \\
\hline 17 & ZINC000150338698 & Capastat & -5.58 & Anti-bacterial- Tuberculosis ${ }^{44}$ \\
\hline 18 & ZINC000000001145 & Ciclopirox & -5.54 & Anti-fungal $^{45}$ \\
\hline 19 & ZINC000100074252 & Sofosbuvir & -5.51 & Hepatitis C Virus RNA polymerase inhibitor ${ }^{46}$ \\
\hline 20 & ZINC000007997568 & Nitrofurantonin & -5.4 & Antibacterial (DNA, RNA and protein inhibition) ${ }^{47}$ \\
\hline
\end{tabular}

\subsection{Induced fit docking}

The initial 20 HTVS hits were further subjected to Glide XP and induced fit docking protocol to

find out the final poses of the drugs with better binding affinity. From the IFD analysis we have identified top 10 compound and their corresponding structures are presented in Figure 1. The docking score of top 10 compounds along with interaction residues are listed in table 2. 


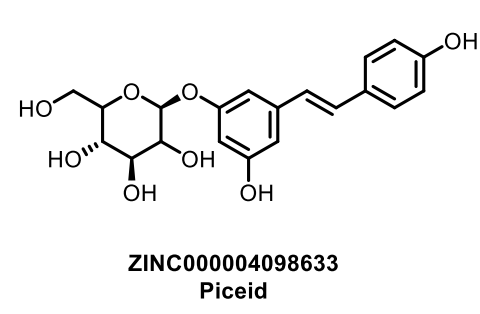

Piceid<smiles>Cn1c(N)c(NC(=O)NCCN)c[n+]1CC1=C(C(=O)[O-])N2C(=O)[C@@H](NC(=O)/C(=N\OC(C)(C)C(=O)O)c3nsc(N)n3)[C@H]2SC1</smiles>

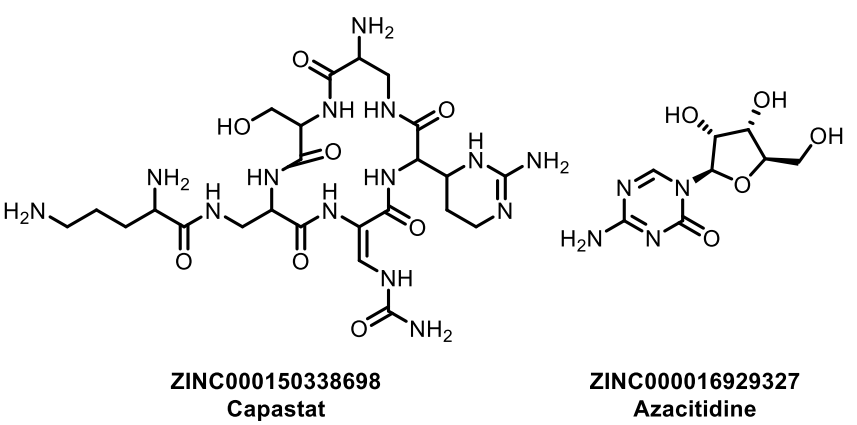

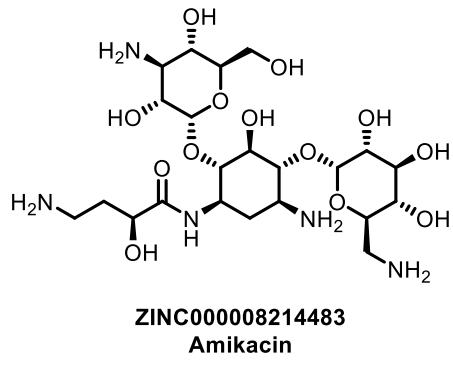

Amikacin<smiles>CN[C@H]1[C@@H](O)[C@H](O[C@H]2C(NC(=O)C(O)CCN)C[C@@H](N)[C@H](O[C@@H]3OC(CNCCO)=CC[C@H]3N)[C@H]2O)OC[C@]1(C)O</smiles>
Plazomicin<smiles>CC(C)OC(=O)[C@H](COP(=O)(Oc1ccccc1)Oc1ccccc1)OC1OC(n2ccc(=O)[nH]c2=O)[C@H](O)[C@@H]1C(F)(F)F</smiles>

ZINC000100074252 Sofosbuvir<smiles>CSCCNc1nc(SCCC(F)(F)F)nc2c1ncn2[C@@H]1OC(COP(=O)(O)OP(=O)(O)C(Cl)(Cl)P(=O)(O)O)[C@@H](O)[C@H]1O</smiles>

ZINC000085537017

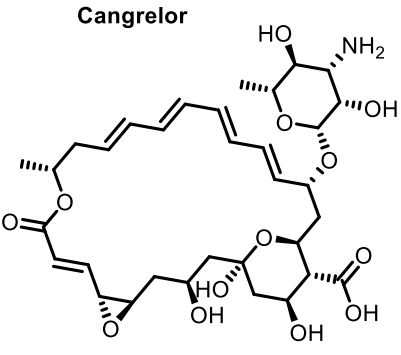

ZINC000253633751

Natamycin

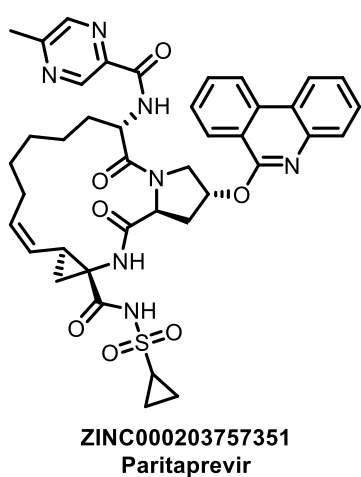

Fig. 1. Chemical structure of top 10 hits from IFD

The IFD analysis reveals piceid (ZINC000004098633) to have excellent binding affinity towards Nsp15 catalytic site (Glide score-11.66 $\mathrm{kcal} / \mathrm{mol}$ ). In the active site piceid is stabilized by hydrogen bonding interactions with GLN245, GLY248, LYS290, SER294, and GLU340 amino acid residues (Figure 2a). Piceid is a stilbenoid glucoside present in grape juice and it is known to have broad spectrum of biological activity such as Anti-arteriosclerosis, anti-proliferative property $^{31}$, anti-inflammatory property, hepatoprotective effect, lung protective effect, neuroprotective properties etc ${ }^{32}$. 

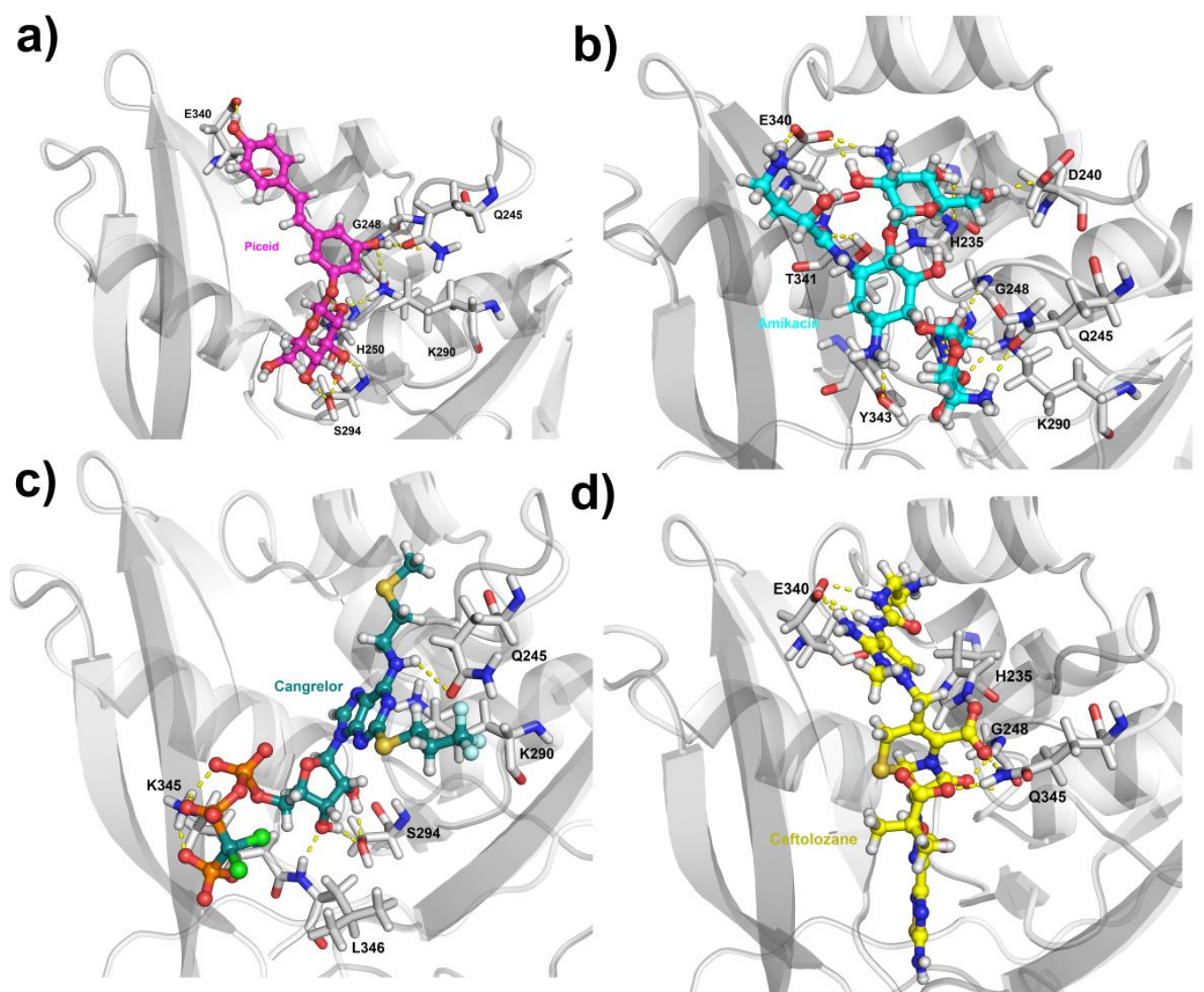

d)
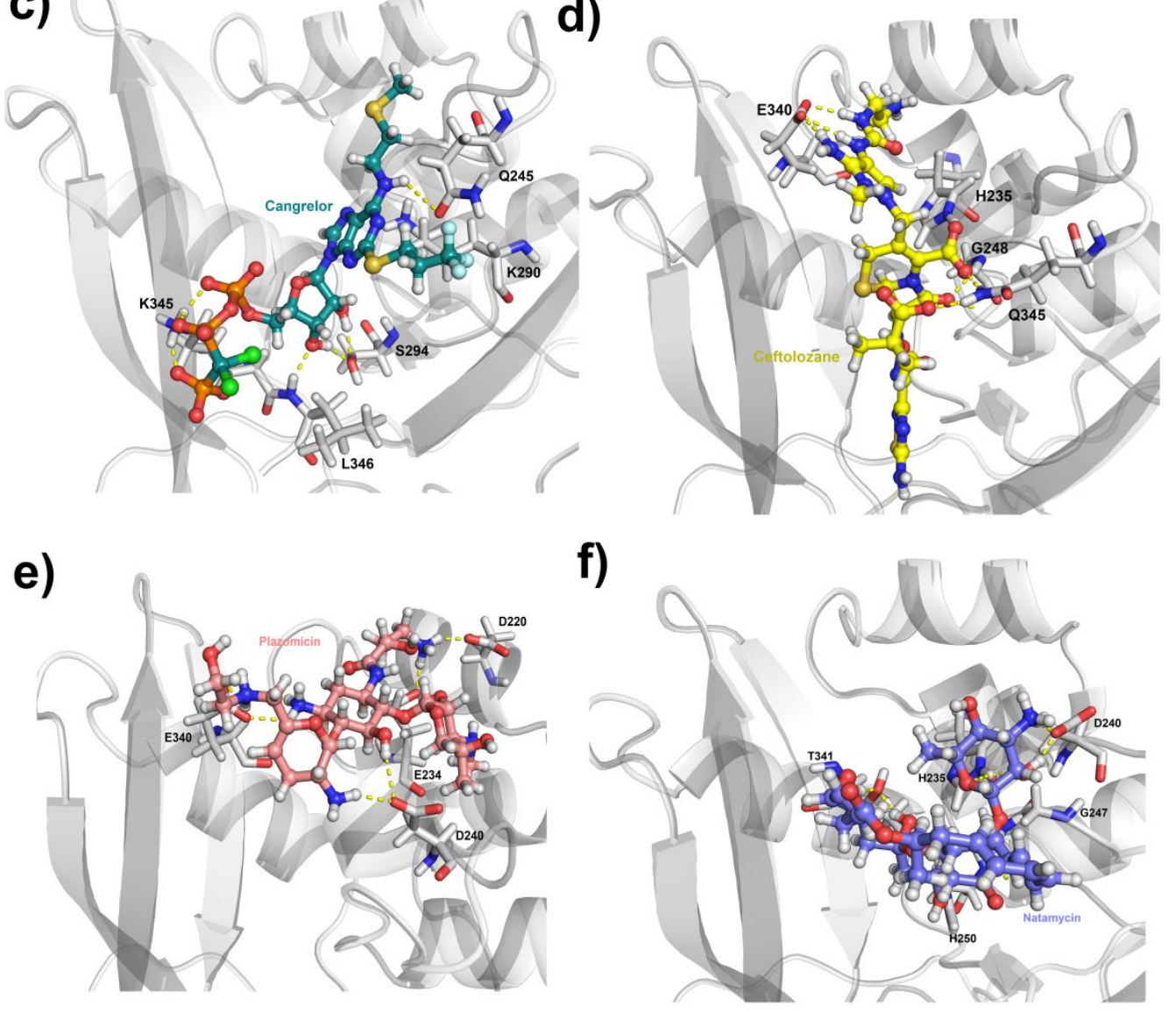

Fig. 2. Final poses of top 10 FDA hits (represented in ball and stick model) with Nsp15 endoribonuclease (represented in grey cartoon and interacting residues in stick model and labelled). a) Piceid b) Amikacin c) Cangrelor d) ceftolozane e) Plazomicin f) Natamycin. All polar contacts are highlighted as yellow dotted lines. 
The next best docking score obtained was for amikacin having $-11.00 \mathrm{kcal} / \mathrm{mol}$. It is an aminoglycoside antibiotic that exhibited several hydrogen bonding interactions with side chain residues ASP240, GLN245, LYS290, GLU340, THR341, and TYR343 in the active site of Nsp15 (Figure 2b). (bond length: 1.9 - 2.4Å). Also, it formed ח...cation interaction with HIS235 residue forming enhanced stabilization. The known inhibitory mechanism of amikacin involves irreversible binding to bacterial 16S rRNA, which prevents the binding of 30 S subunit of prokaryotic ribosome. Thereby, the protein synthesis of the bacteria is halted; amikacin serves as an effective antibiotic against several drug resistant bacteria. Cangrelor is a blood platelet inhibitor (P2Y12 receptor) used for treating coronary artery diseases. It showed docking score of $-10.00 \mathrm{kcal} / \mathrm{mol}$ contributed by hydrogen bonding interaction with ASP240, GLN245, GLY248, LYS290, GLU340, THR341, and TYR343 (Figure 2c). Also, a phosphate moiety in cangrelor forms a salt bridge with side chain of residue LYS345 which provides extra stability to the protein-ligand complex.

Ceftolozane is a $\beta$-lactum antibiotic effective against gram negative bacteria. This drug is currently prescribed for complicated urinary tract infections, intra-abdominal infections, and bacterial pneumonia. A docking score of $-9.67 \mathrm{kcal} / \mathrm{mol}$ was obtained for ceftolozane through the $\mathrm{H}$-bond interactions with side chain and back bone residues (HIS235, GLN245, GLY248, LYS290, GLU340) of Nsp15 (Figure 2d). Further, the imidazole ring of ceftolozane showed edge to face $\pi \ldots \pi$ interaction with TRP333 helps in improving the docking score. Plazomicin is another aminoglycoside antibiotic used for treating gram negative bacterial infections (urinary tract infections). This is the fifth best compound in IFD against Nsp15 with docking score of $9.25 \mathrm{kcal} / \mathrm{mol}$. Like amikacin it also has shown hydrogen bonding interactions with side chains of residues ASP220, GLU234, HIS235, and ASP240 (Figure 2e). Besides, it formed a salt bridge as well as a $\pi$...cation interaction with GLU340 and HIS 235 respectively. 
Natamycin and capastat are the $6^{\text {th }}$ and $7^{\text {th }}$ best compounds in our IFD analysis with the docking score of -8.69 and $-8.4 \mathrm{kcal} / \mathrm{mol}$ (Figure $2 \mathrm{f}$ and Figure $3 \mathrm{a}$ for natamycin and capastat respectively). Azacitidine and oteracil are the anti-cancer drugs with docking scores of -7.27 and $-6.23 \mathrm{kcal} / \mathrm{mol}$ respectively (Figure $3 \mathrm{~b}$ ). It is noteworthy that azacitidine is potential inhibitor against HIV and human T-lymphotropic virus. Among the antiviral drugs (Saquinavir, Paritaprevir Dolutegravir, Sofosbuvir), sofosbuvir and paritaprevir showed higher binding affinity with the docking scores of -6.75 and $-6.19 \mathrm{kcal} / \mathrm{mol}$ respectively (Figure $3 \mathrm{c}$ and d).

a)

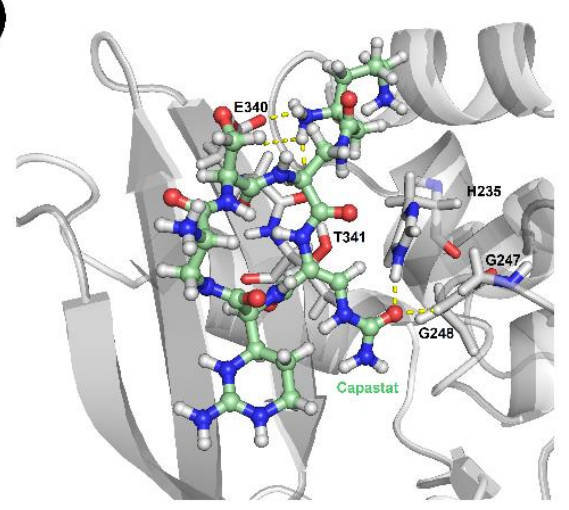

c)

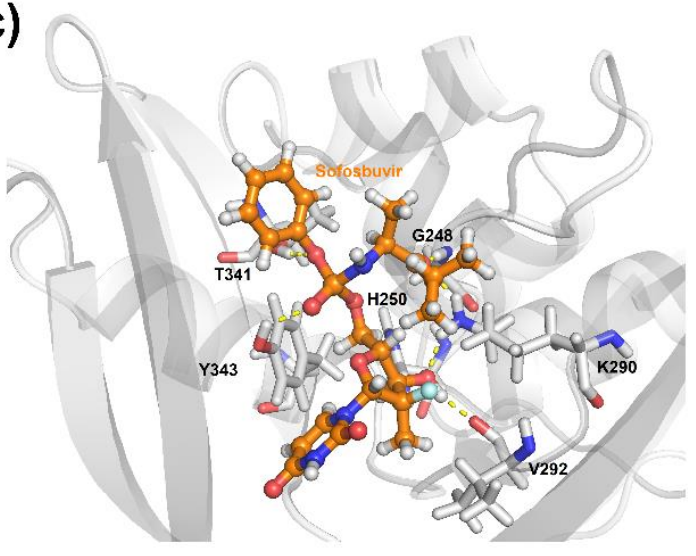

b)

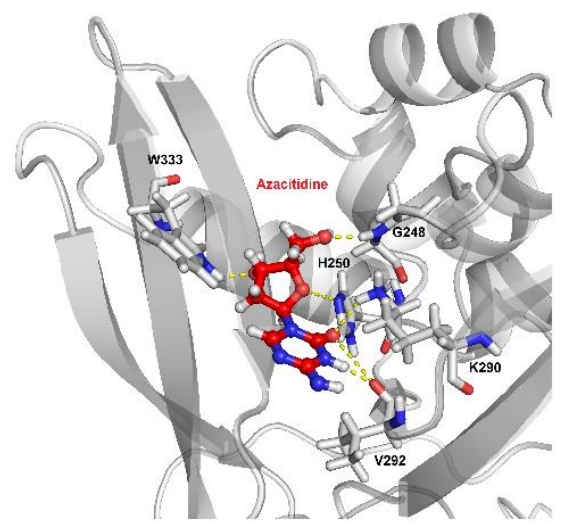

d)

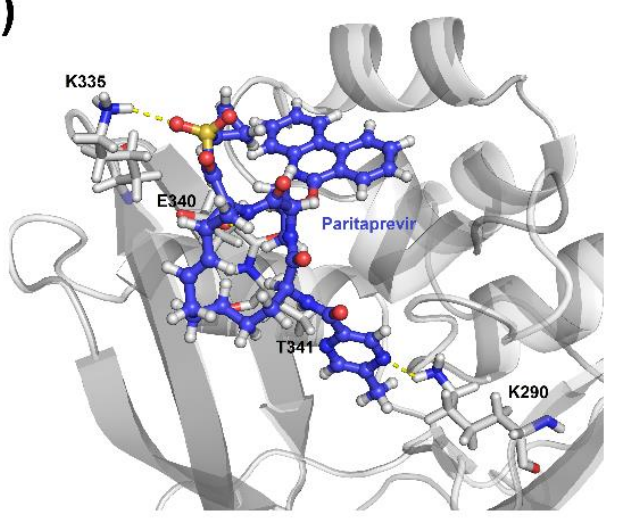

Fig. 3. Final poses of top 10 FDA hits (represented in ball and stick model) with Nsp15 endoribonuclease (represented in grey cartoon, interacting residues in stick model and labelled). a) Capastat b) Azacitidine c) Sofosbuvir d) Paritaprevir. All polar contacts are highlighted as yellow dotted lines. 
Table 2. Docking score of the virtual screening hits after IFD protocol.

\begin{tabular}{|c|c|c|c|c|c|}
\hline S.no & Compound ID & $\begin{array}{l}\text { Compound } \\
\text { name }\end{array}$ & Glide score & $\begin{array}{l}\text { No.of H-bond } \\
\text { interactions }\end{array}$ & Interacting residues \\
\hline 1 & ZINC000004098633 & Piceid & -11.664 & 6 & $\begin{array}{c}\text { H-bonding (GLN245, GLY248, LYS290, SER294, } \\
\text { and GLU340) }\end{array}$ \\
\hline 2 & ZINC000008214483 & Amikacin & -11.005 & 10 & $\begin{array}{c}\text { H-bonding ASP240, GLN245, GLY248, LYS290, } \\
\text { GLU340, THR341, and TYR343) п....cation } \\
\text { interaction (HIS235) }\end{array}$ \\
\hline 3 & ZINC000085537017 & Cangrelor & -10.003 & 6 & $\begin{array}{c}\text { H-bonding (GLN245, LYS290, SER294, LEU346, } \\
\text { LYS345) Salt bridges (LYS345) }\end{array}$ \\
\hline 4 & ZINC000009164421 & Ceftolozane & -9.678 & 6 & $\begin{array}{c}\text { H-bonding (HIS235, GLN245, GLY248, LYS290, } \\
\text { GLU340) and } \pi . . . \pi \text { (TRP333) }\end{array}$ \\
\hline 5 & ZINC000068150640 & Plazomicin & -9.251 & 5 & $\begin{array}{l}\text { H-bonding (ASP220, GLU234, HIS235, ASP240) } \\
\text { Salt bridges (GLU340) and m...cation (HIS235) }\end{array}$ \\
\hline 6 & ZINC000253633751 & Natamycin & -8.695 & 4 & $\begin{array}{c}\text { H-bonding (GLY248, LYS335, GLU340, THR 341) } \\
\text { and } \pi \ldots \pi(\mathrm{HIS} 235 \text { and TYR343) }\end{array}$ \\
\hline 7 & ZINC000150338698 & Capastat & -8.400 & 3 & $\begin{array}{l}\text { H-bonding (HIS235, GLY248, and GLU340) } \\
\text { Salt bridges (GLU234 and GLU340) }\end{array}$ \\
\hline 8 & ZINC000016929327 & Azacitidine & -7.270 & 3 & H-bonding (LYS290, VAL292) \\
\hline 9 & ZINC000100074252 & Sofosbuvir & -6.753 & 6 & $\begin{array}{c}\text { H-bonding (LEU246, GLY248, LYS290, VAL292, } \\
\text { THR341, THR343) }\end{array}$ \\
\hline 10 & ZINC000203757351 & Paritaprevir & -6.199 & 4 & $\begin{array}{c}\text { H-bonding (GLY248, LYS335, GLU340 THR341) } \\
\text { п... m interaction (HIS235 and THR343) }\end{array}$ \\
\hline
\end{tabular}

\subsection{Molecular Dynamics (MD) simulation study}

The stability of the top five compounds along with Nsp15 Endoribonuclease from SARS CoV-2 was evaluated by using molecular dynamic simulations. The duration of MD was $10 \mathrm{~ns}$ for each protein-ligand complex in TIP3P water system. The c-alpha atom Root mean square Deviation (RMSD) of NSP15 and ligand RMSD plots were represented in Figure 4.

Piceid was the best docked drug with Nsp15 endoribonuclease in IFD analysis. The Nsp15 residues that are involved in hydrogen bonding with piceid in our molecular dynamic simulation are GLY248, HIS250, LYS290, SER294. TYR343 strongly stabilizes the protein ligand complex by hydrophobic and water bridges interactions. The C-alpha RMSD of Nsp15 with ZINC000004098633 (Piceid) which reached the maximum of $2.4 \AA$ at around 1 ns and was 
stable throughout the course of simulation. (Figure $4 a$ ). Figure $4 b$ represents the C-alpha RMSD of Nsp15 with ZINC000008214483 (Amikacin) which reached the maximum of $3.2 \AA$ at around 4 ns and the average RMSD was found to be $2.0 \AA$ throughout the course of simulation. GLU340, a key residue of Nsp15 forms a hydrogen bonding interaction with the amino group of amikacin and this interaction was observed to be stable throughout the duration of MD simulation.

Figure 4c represents the RMSD of Nsp15 with ZINC000085537017 (Cangrelor) which reached the maximum of $2.1 \AA$ at around 3 ns and was stable throughout the course of simulation. LYS345, LYS290, SER294, TYR343, PRO344, LEU346 and GLN347 are the main residue of Nsp15 facilitating interactions with ZINC000085537017. Also, Nsp15 and cangrelor interaction was mediated by water bridges with the help of residues PRO344, LEU346 and GLN347. The RMSD of Nsp15 with ZINC000009164421 (Ceftolozane) reached the maximum of $2.8 \AA$ at 4 ns. The average RMSD for the entire simulation was stabilized at $2.4 \AA$. The Protein-ligand interactions were monitored throughout the simulation and represented in Figure 4d. LYS290, GLU340, TYR343, GLY248, and HIS235 are the main residues of Nsp15 facilitating interactions with ceftolozane. Figure 4e presents the RMSD of Nsp15 with ZINC000068150640 (Plazomicin) reached maximum of $2.7 \AA$ around at $2 \mathrm{~ns}$ and then showed average RMSD of $1.8 \AA$ throughout the course of simulation. GLU234, GLU229 and GLU340 in the a-helix and loops are the major interacting residues of Nsp15 with plazomicin.

Similarly, MD simulations for sofosbuvir and azacitidine individually complexed with Nsp15 were performed for $10 \mathrm{~ns}$, which were observed to be quite stable. And the resultant data for these compounds are provided in the supplementary figure $1 \mathrm{~S}$ and $2 \mathrm{~S}$. These results suggest that the above compounds can be explored against Nsp15 endoribonuclease from SARS CoV-2. Further, the other compounds present in top 20 has also demonstrated affinity towards the Nsp15 catalytic triad and can serve as a potential lead or chemical scaffolds to understand the 
rationale behind the development of novel Nsp15 inhibitors. However, further experimental studies are required to elucidate the above said hypothesis.

a)

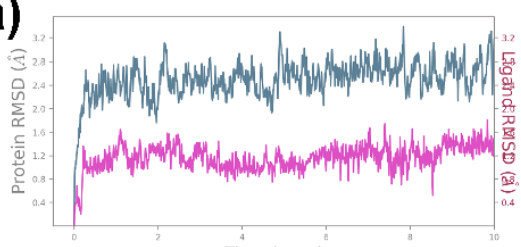

b)
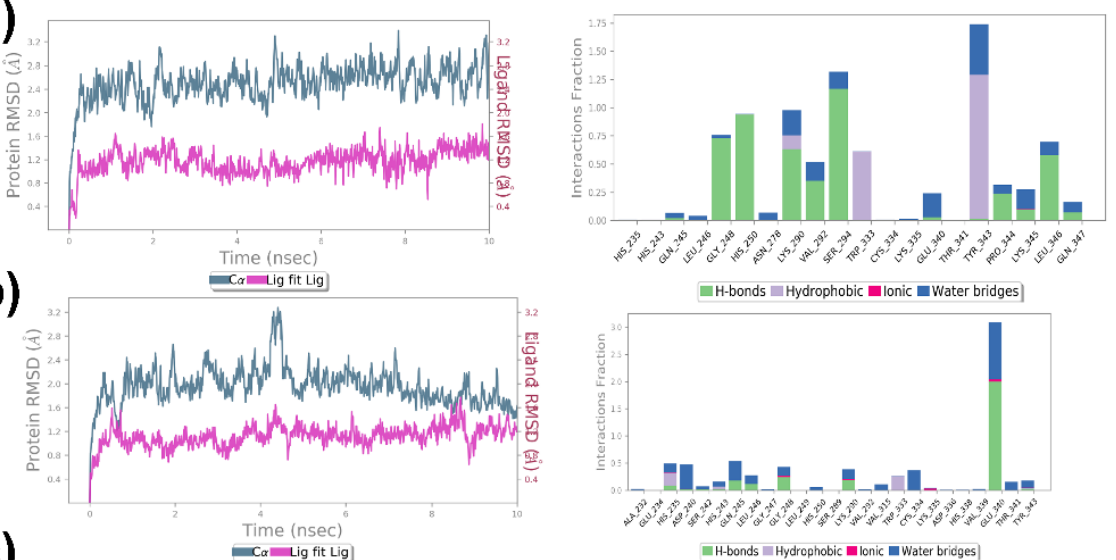

c)
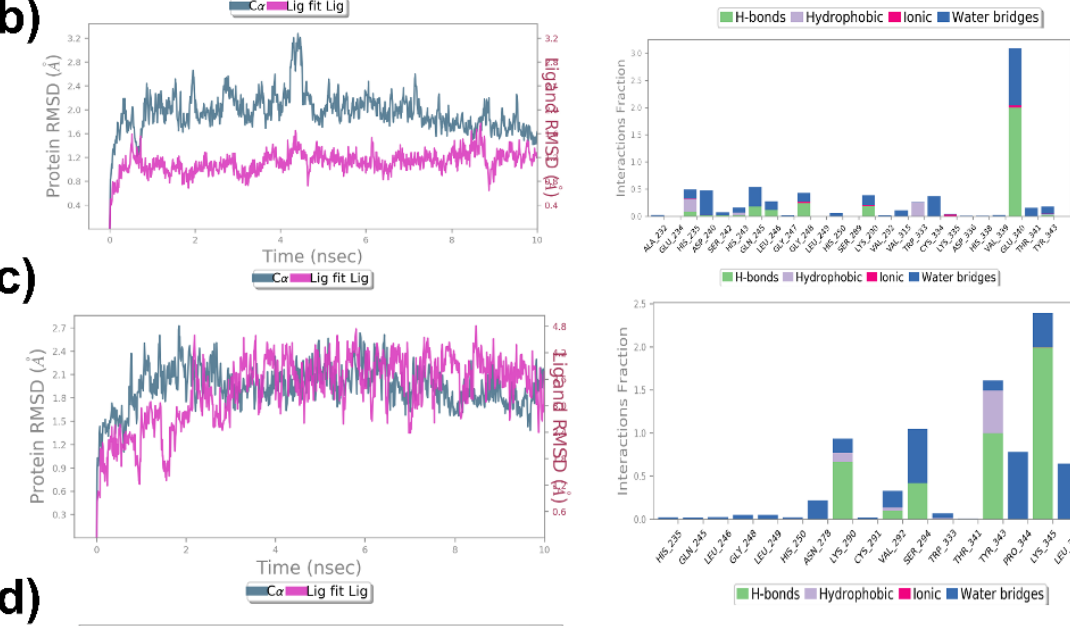

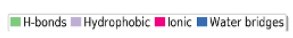
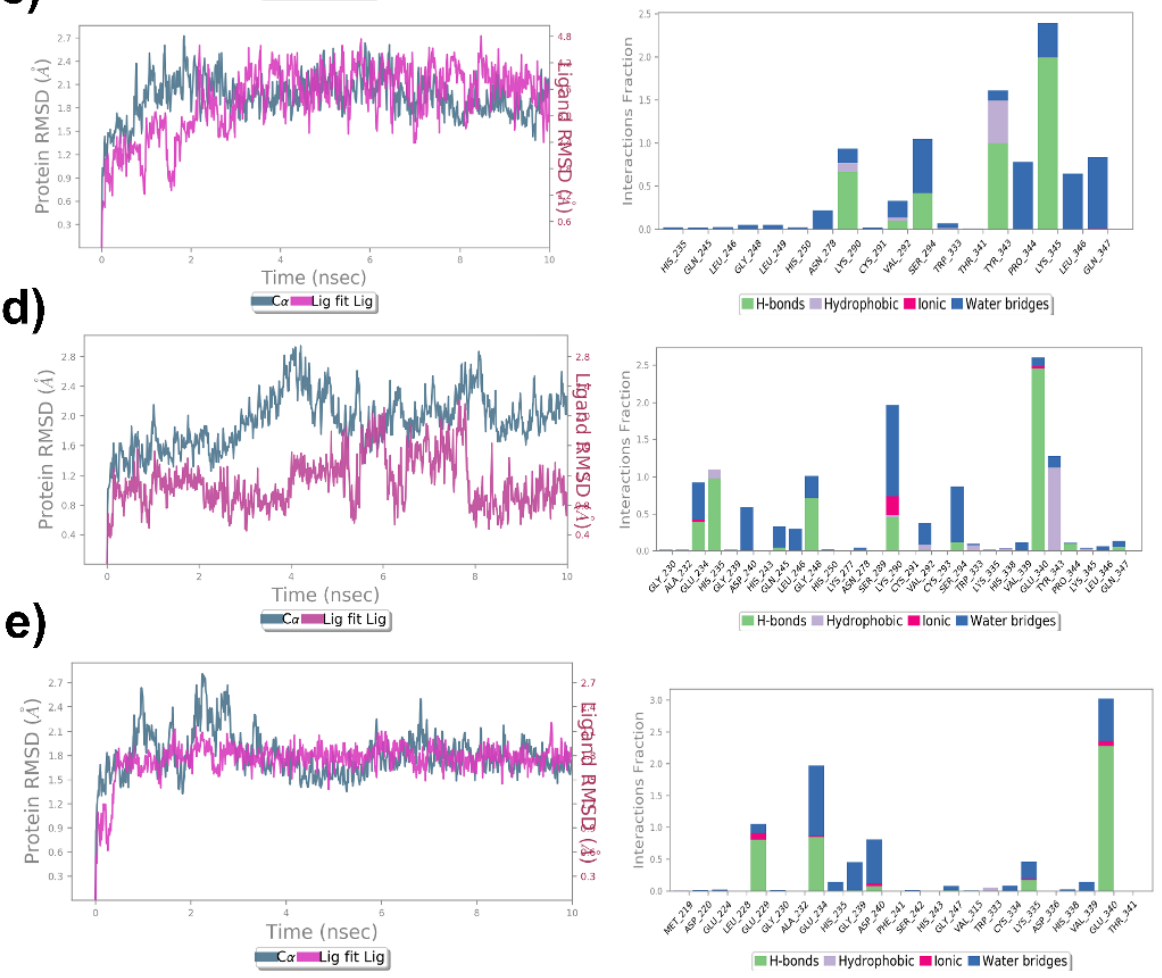

Fig. 4. The c-alpha atom RMSD (represented in blue) of NSP15 and ligand RMSD (represented in magenta) plots are presented on left side of the figure. Also, protein interactions with ligands was monitored throughout the $10 \mathrm{~ns}$ simulation and represented on right side of the figure (protein - ligand interactions categorized as $\mathrm{H}$-bonds, hydrophobic, ionic and water bridges). 


\section{Conclusion}

In the biological system, Nsp15 is a hexameric endoribonuclease that helps in Coronavirus genomic RNA replication. Both structurally and functionally SARS-CoV2 Nsp15 is quite like SARS-CoV Nsp15. Therefore, the inhibitors designed for SARS-CoV Nsp15 should work against SARS-CoV2 Nsp15. Nsp15 is highly conserved and the fact that the active enzyme is a hexamer makes it an interesting target. Reusing the existing drugs can save time, money and most of all many lives. However, methodological means of finding an effective pre-existing drug is mandatory to prevent any undesirable effects. In this study, we have performed virtual screening of 2910 FDA drugs against Nsp15 Endoribonuclease from SARS CoV2 and isolated the top 20 compounds for the study. The top 20 hits which had better interaction energy with Nsp15 were further subjected to IFD protocol to find out the final pose of the drugs. Through IFD studies, we found that Piceid, Amikacin, cangrelor, ceftolozane and plazomicin have very strong interaction with Nsp15. The final protein-ligand pose/docking pose of the 8 compounds were simulated for $10 \mathrm{~ns}$ and was found to be stable throughout the course of simulation. Hydrogen bonding, $\pi \ldots \pi$, water bridges and $\pi$...cation interactions are important for affinity towards Nsp15. From our in-silico docking and MD studies, we have shown that picied, amikacin, cangrelor, ceftolozane and plazomicin drugs can inhibit Nsp15 and might be of value for treating COVID-19. However, further experimental studies are required to elucidate on this hypothesis.

\section{Acknowledgements}

The authors would like to thank Indian Institute of Technology Gandhinagar for infrastructure. VT and SK thanks DST-SERB for funding.

\section{References}

1. Cui, J.; Li, F.; Shi, Z.-L., Origin and evolution of pathogenic coronaviruses. Nat Rev Microbiol 2019, 17 (3), 181-192.

2. Lu, R.; Zhao, X.; Li, J.; Niu, P.; Yang, B.; Wu, H.; Wang, W.; Song, H.; Huang, B.; Zhu, N.; Bi, Y.; Ma, X.; Zhan, F.; Wang, L.; Hu, T.; Zhou, H.; Hu, Z.; Zhou, W.; Zhao, L.; Chen, J.; Meng, Y.; Wang, J.; Lin, Y.; 
Yuan, J.; Xie, Z.; Ma, J.; Liu, W. J.; Wang, D.; Xu, W.; Holmes, E. C.; Gao, G. F.; Wu, G.; Chen, W.; Shi, W.; Tan, W., Genomic characterisation and epidemiology of 2019 novel coronavirus: implications for virus origins and receptor binding. The Lancet 2020, 395 (10224), 565-574.

3. Morse, J. S.; Lalonde, T.; Xu, S.; Liu, W. R., Learning from the Past: Possible Urgent Prevention and Treatment Options for Severe Acute Respiratory Infections Caused by 2019-nCoV. Chembiochem 2020, 21 (5), 730-738.

4. Wrapp, D.; Wang, N.; Corbett, K. S.; Goldsmith, J. A.; Hsieh, C.-L.; Abiona, O.; Graham, B. S.; McLellan, J. S., Cryo-EM structure of the 2019-nCoV spike in the prefusion conformation. Science (New York, N.Y.) 2020, 367 (6483), 1260-1263.

5. $\quad$ Du, L.; He, Y.; Zhou, Y.; Liu, S.; Zheng, B.-J.; Jiang, S., The spike protein of SARS-CoV - a target for vaccine and therapeutic development. Nature Reviews Microbiology 2009, 7 (3), 226-236.

6. Shang, J.; Ye, G.; Shi, K.; Wan, Y.; Luo, C.; Aihara, H.; Geng, Q.; Auerbach, A.; Li, F., Structural basis of receptor recognition by SARS-CoV-2. Nature 2020.

7. Liu, C.; Zhou, Q.; Li, Y.; Garner, L. V.; Watkins, S. P.; Carter, L. J.; Smoot, J.; Gregg, A. C.; Daniels, A. D.; Jervey, S.; Albaiu, D., Research and Development on Therapeutic Agents and Vaccines for COVID19 and Related Human Coronavirus Diseases. ACS Central Science 2020, 6 (3), 315-331.

8. Nedialkova, D. D.; Ulferts, R.; van den Born, E.; Lauber, C.; Gorbalenya, A. E.; Ziebuhr, J.; Snijder, E. J., Biochemical characterization of arterivirus nonstructural protein 11 reveals the nidovirus-wide conservation of a replicative endoribonuclease. Journal of virology 2009, 83 (11), 5671-82.

9. Bhardwaj, K.; Sun, J.; Holzenburg, A.; Guarino, L. A.; Kao, C. C., RNA recognition and cleavage by the SARS coronavirus endoribonuclease. Journal of molecular biology 2006, 361 (2), 243-56.

10. Knoops, K.; Kikkert, M.; Worm, S. H.; Zevenhoven-Dobbe, J. C.; van der Meer, Y.; Koster, A. J.; Mommaas, A. M.; Snijder, E. J., SARS-coronavirus replication is supported by a reticulovesicular network of modified endoplasmic reticulum. PLoS biology 2008, 6 (9), e226.

11. Deng, X.; Hackbart, M.; Mettelman, R. C.; O'Brien, A.; Mielech, A. M.; Yi, G.; Kao, C. C.; Baker, S. C., Coronavirus nonstructural protein 15 mediates evasion of dsRNA sensors and limits apoptosis in macrophages. Proc Natl Acad Sci U S A 2017, 114 (21), E4251-e4260.

12. Bhardwaj, K.; Guarino, L.; Kao, C. C., The Severe Acute Respiratory Syndrome Coronavirus Nsp15 Protein Is an Endoribonuclease That Prefers Manganese as a Cofactor. Journal of Virology 2004, 78 (22), 12218-12224.

13. Zheng, A.; Shi, Y.; Shen, Z.; Wang, G.; Shi, J.; Xiong, Q.; Fang, L.; Xiao, S.; Fu, Z. F.; Peng, G., Insight into the evolution of nidovirus endoribonuclease based on the finding that $n s p 15$ from porcine Deltacoronavirus functions as a dimer. The Journal of biological chemistry 2018, 293 (31), 12054-12067. 14. Ivanov, K. A.; Hertzig, T.; Rozanov, M.; Bayer, S.; Thiel, V.; Gorbalenya, A. E.; Ziebuhr, J., Major genetic marker of nidoviruses encodes a replicative endoribonuclease. Proceedings of the National Academy of Sciences of the United States of America 2004, 101 (34), 12694-12699.

15. Korber, B.; Fischer, W.; Gnanakaran, S. G.; Yoon, H.; Theiler, J.; Abfalterer, W.; Foley, B.; Giorgi, E. E.; Bhattacharya, T.; Parker, M. D.; Partridge, D. G.; Evans, C. M.; de Silva, T.; LaBranche, C. C.; Montefiori, D. C., Spike mutation pipeline reveals the emergence of a more transmissible form of SARSCoV-2. bioRxiv 2020, 2020.04.29.069054.

16. Bhardwaj, K.; Palaninathan, S.; Alcantara, J. M. O.; Li Yi, L.; Guarino, L.; Sacchettini, J. C.; Kao, C. C., Structural and Functional Analyses of the Severe Acute Respiratory Syndrome Coronavirus Endoribonuclease Nsp15. Journal of Biological Chemistry 2008, 283 (6), 3655-3664.

17. Kim, Y.; Jedrzejczak, R.; Maltseva, N. I.; Endres, M.; Godzik, A.; Michalska, K.; Joachimiak, A., Crystal structure of Nsp15 endoribonuclease NendoU from SARS-CoV-2. bioRxiv 2020, 2020.03.02.968388.

18. Berman, H. M.; Westbrook, J.; Feng, Z.; Gilliland, G.; Bhat, T. N.; Weissig, H.; Shindyalov, I. N.; Bourne, P. E., The Protein Data Bank. Nucleic Acids Research 2000, 28 (1), 235-242. 
19. Irwin, J. J.; Shoichet, B. K., ZINC--a free database of commercially available compounds for virtual screening. Journal of chemical information and modeling 2005, 45 (1), 177-82.

20. Ortiz-Alcantara, J.; Bhardwaj, K.; S, P.; et, a., Small molecule inhibitors of the SARS-CoV Nsp15 endoribonuclease. Virus Adaptation and Treatment 2010, 2.

21. Repasky, M. P.; Shelley, M.; Friesner, R. A., Flexible ligand docking with Glide. Current protocols in bioinformatics 2007, Chapter 8, Unit 8.12.

22. Dapp, M. J.; Clouser, C. L.; Patterson, S.; Mansky, L. M., 5-Azacytidine Can Induce Lethal Mutagenesis in Human Immunodeficiency Virus Type 1. Journal of Virology 2009, 83 (22), 11950-11958.

23. Diamantopoulos, P. T.; Michael, M.; Benopoulou, O.; Bazanis, E.; Tzeletas, G.; Meletis, J.; Vayopoulos, G.; Viniou, N. A., Antiretroviral activity of 5-azacytidine during treatment of a HTLV-1 positive myelodysplastic syndrome with autoimmune manifestations. Virology journal 2012, 9, 1.

24. Sorbera, M.; Chung, E.; Ho, C. W.; Marzella, N., Ceftolozane/Tazobactam: a new option in the treatment of complicated gram-negative infections. P T 2014, 39 (12), 825-832.

25. Long, T. E.; Williams, J. T., Cephalosporins currently in early clinical trials for the treatment of bacterial infections. Expert Opinion on Investigational Drugs 2014, 23 (10), 1375-1387.

26. Estey, E. H., Epigenetics in clinical practice: the examples of azacitidine and decitabine in myelodysplasia and acute myeloid leukemia. Leukemia 2013, 27 (9), 1803-12.

27. Cheloff, A. Z.; Al-Samkari, H., Avatrombopag for the treatment of immune thrombocytopenia and thrombocytopenia of chronic liver disease. J Blood Med 2019, 10, 313-321.

28. Bjelogrlic, S. K.; Radic, J.; Radulovic, S.; Jokanovic, M.; Jovic, V., Effects of dexrazoxane and amifostine on evolution of Doxorubicin cardiomyopathy in vivo. Experimental biology and medicine (Maywood, N.J.) 2007, 232 (11), 1414-24.

29. Loyevsky, M.; Sacci, J. B., Jr.; Boehme, P.; Weglicki, W.; John, C.; Gordeuk, V. R., Plasmodium falciparum and Plasmodium yoelii: effect of the iron chelation prodrug dexrazoxane on in vitro cultures. Experimental parasitology 1999, 91 (2), 105-14.

30. Peters, G. J.; Noordhuis, P.; Van Kuilenburg, A. B.; Schornagel, J. H.; Gall, H.; Turner, S. L.; Swart, M. S.; Voorn, D.; Van Gennip, A. H.; Wanders, J.; Holwerda, U.; Smid, K.; Giaccone, G.; Fumoleau, P.; Van Groeningen, C. J., Pharmacokinetics of S-1, an oral formulation of ftorafur, oxonic acid and 5-chloro-2,4dihydroxypyridine (molar ratio 1:0.4:1) in patients with solid tumors. Cancer chemotherapy and pharmacology 2003, 52 (1), 1-12.

31. Storniolo, C. E.; Quifer-Rada, P.; Lamuela-Raventos, R. M.; Moreno, J. J., Piceid presents antiproliferative effects in intestinal epithelial Caco-2 cells, effects unrelated to resveratrol release. Food \& function 2014, 5 (9), 2137-44.

32. Du, Q.-H.; Peng, C.; Zhang, H., Polydatin: A review of pharmacology and pharmacokinetics. Pharmaceutical Biology 2013, 51 (11), 1347-1354.

33. Kubica, J.; Kozinski, M.; Navarese, E. P.; Tantry, U.; Kubica, A.; Siller-Matula, J. M.; Jeong, Y. H.; Fabiszak, T.; Andruszkiewicz, A.; Gurbel, P. A., Cangrelor: an emerging therapeutic option for patients with coronary artery disease. Current medical research and opinion 2014, 30 (5), 813-28.

34. te Welscher, Y. M.; ten Napel, H. H.; Balagué, M. M.; Souza, C. M.; Riezman, H.; de Kruijff, B.; Breukink, E., Natamycin blocks fungal growth by binding specifically to ergosterol without permeabilizing the membrane. The Journal of biological chemistry 2008, 283 (10), 6393-401.

35. Eljaaly, K.; Alharbi, A.; Alshehri, S.; Ortwine, J. K.; Pogue, J. M., Plazomicin: A Novel Aminoglycoside for the Treatment of Resistant Gram-Negative Bacterial Infections. Drugs 2019, 79 (3), 243-269.

36. Wagenlehner, F. M. E.; Cloutier, D. J.; Komirenko, A. S.; Cebrik, D. S.; Krause, K. M.; Keepers, T. R.; Connolly, L. E.; Miller, L. G.; Friedland, I.; Dwyer, J. P., Once-Daily Plazomicin for Complicated Urinary Tract Infections. New England Journal of Medicine 2019, 380 (8), 729-740. 
37. Dasso, L.; Al-Khaled, T.; Sonty, S.; Aref, A. A., Profile of netarsudil ophthalmic solution and its potential in the treatment of open-angle glaucoma: evidence to date. Clin Ophthalmol 2018, 12, 19391944.

38. Hong, L. I. N.; Zhang, X. C.; Hartsuck, J. A.; Tang, J., Crystal structure of an in vivo HIV-1 protease mutant in complex with saquinavir: Insights into the mechanisms of drug resistance. Protein Science 2000, 9 (10), 1898-1904.

39. Alsenz, J.; Steffen, H.; Alex, R., Active Apical Secretory Efflux of the HIV Protease Inhibitors Saquinavir and Ritonavir in Caco-2 Cell Monolayers. Pharmaceutical Research 1998, 15 (3), 423-428. 40. HARRISON, D. C.; CHIDSEY, C. A.; BRAUNWALD, E., Studies on the Mechanism of Action of Metaraminol (Aramine). Annals of Internal Medicine 1963, 59 (3), 297-305.

41. Price, K. E.; DeFuria, M. D.; Pursiano, T. A., Amikacin, an Aminoglycoside with Marked Activity against Antibiotic-Resistant Clinical Isolates. The Journal of Infectious Diseases 1976, 134 (Supplement_2), S249-S261.

42. Paritaprevir/ritonavir/ombitasvir plus dasabuvir with ribavirin for chronic hepatitis C. Aust Prescr 2016, 39 (4), 141-143.

43. Kandel, C. E.; Walmsley, S. L., Dolutegravir - a review of the pharmacology, efficacy, and safety in the treatment of HIV. Drug Des Devel Ther 2015, 9, 3547-3555.

44. Lin, Y.; Li, Y.; Zhu, N.; Han, Y.; Jiang, W.; Wang, Y.; Si, S.; Jiang, J., The Antituberculosis Antibiotic Capreomycin Inhibits Protein Synthesis by Disrupting Interaction between Ribosomal Proteins L12 and L10. Antimicrobial Agents and Chemotherapy 2014, 58 (4), 2038-2044.

45. Leem, S. H.; Park, J. E.; Kim, I. S.; Chae, J. Y.; Sugino, A.; Sunwoo, Y., The possible mechanism of action of ciclopirox olamine in the yeast Saccharomyces cerevisiae. Molecules and cells 2003, 15 (1), 5561.

46. Bhatia, H. K.; Singh, H.; Grewal, N.; Natt, N. K., Sofosbuvir: A novel treatment option for chronic hepatitis C infection. J Pharmacol Pharmacother 2014, 5 (4), 278-284.

47. McOsker, C. C.; Fitzpatrick, P. M., Nitrofurantoin: mechanism of action and implications for resistance development in common uropathogens. The Journal of antimicrobial chemotherapy 1994, 33 Suppl A, 23-30. 